\title{
Les facteurs angiogéniques
}

Des découvertes récentes ont encore avivé l'intérêt porté aux facteurs de l'angiogenèse [1, 2]. Ceux-ci interviennent en effet dans deux types d'événements d'importance capitale : chez l'individu normal, ils assurent la formation des vaisseaux sanguins lors des processus de réparation, mais aussi dans des situations physiologiques comme l'ovulation; en pathologie, leur rôle le plus spectaculaire s'exerce sur le développement des tumeurs : suivant les cas, ils en limitent ou en favorisent la croissance, ce qui a donné naissance à l'hypothèse que les tumeurs solides seraient dépendantes de l'angiogenèse, avec les conséquences thérapeutiques qui pourraient en découler. Pour donner tous ses fruits, l'étude de l'angiogenèse devait se forger des méthodes appropriées ; ces méthodes s'appliquent in vivo, comme la mesure de la croissance des capillaires après implantation dans la cornée du rat ou du lapin, ou l'examen de la membrane chorio-allantoïque de l'embryon de poulet. In vitro, on utilise la culture de cellules endothéliales vasculaires, dont on peut mesurer la locomotion ou, surtout, la prolifération.

A l'heure actuelle, six facteurs ont été identifiés avec certitude: les facteurs de croissance des fibroblastes acide et basique (FGF a et b), l'angiogénine, les facteurs dits de croissance transformants (TGF $\alpha$ et $\beta$ ), et le facteur nécrosant des tumeurs (TNF $\alpha)$. La nomenclature, basée sur la première propriété trouvée dans chaque cas, devra un jour être révisée.

Les FGF ont été les premiers décrits ; à partir de 1983 leur purification est devenue facile grâce à la découverte de leur affinité pour l'héparine [3]. Des clones d'ADNc[1] ont montré que les deux facteurs $a$ et $b$ avaient une chaîne de 154 acides aminés, scindée par protéolyse pour donner les formes de 140 acides aminés pour identiques, leur homologie est d'environ $50 \%$. Leur dénomination tient à leurs $\mathrm{pHi}$, voisins de $\mathrm{pH} 6$ et 9 respectivement. Le FGF acide est surtout concentré dans les tissus nerveux, alors que la distribution du FGF basique est beaucoup plus large. Les cellules contiennent des récepteurs aux FGF, dont il n'est pas établi s'ils diffèrent pour les deux types de facteurs. Les FGF sont actifs in vitro comme in vivo. Des cellules normales en contiennent mais ne les sécrètent pas à l'extérieur, sans doute du fait de l'absence de peptide signal. Leur libération n'a lieu que dans des circonstances particulières, physiologiques comme l'ovulation, pathologiques comme l'attrition des cellules, d'où leur rôle invoqué dans la réparation tissulaire.

L'angiogénine $\left(m / s \quad n^{\circ} 2\right.$, vol. 2, p. 107). Ce polypeptide d'une taille de 14400 Da a été isolé par l'équipe de B. Vallee en 1985 et son gène cloné à partir d'une banque d'ADNc de foie humain. Elle est donc présente dans des tissus normaux, bien qu'elle soit plus abondante dans les tissus cancéreux. Plusieurs caractères l'opposent aux FGF : il n'existe avec eux aucune homologie; elle n'a pas d'affinité pour l'héparine; elle possède un peptide signal et est sécrétée dans le milieu par des cellules en culture ; le fait essentiel, que nous retrouverons pour d'autres facteurs, est le paradoxe apparent de l'opposition entre son comportement in vivo et in vitro: alors qu'elle est extrêmement active sur la vascularisation dans le test de la cornée du lapin, elle n'a aucun effet sur la multiplication in vitro des cellules endothéliales.

Les TGF (transforming growth factors). Ce sont des polypeptides capables d'altérer le phénotype de certaines cellules; en leur présence, des fibroblastes s'immortalisent mais ne deviennent pas cancéreux. On en a découvert deux, dont l'homonymie ne doit pas masquer les différences.
Ils diffèrent quant à leur nature : le TGF $\alpha$ est un peptide de 50 acides aminés synthétisé par les cellules transformées. Il possède une homologie partielle avec l'EGF (epidermal growth factor), qui présente d'ailleurs, à un moindre degré, les mêmes propriétés [4] et se lie au récepteur de l'EGF. Le TGF $\beta$ est un homodimère dont la sous-unité compte 112 acides aminés, provenant de la scission d'un précurseur de 391 acides aminés [5]. On le trouve à la fois dans les tumeurs et dans de nombreux tissus normaux (m/s $n^{\circ} 8$, vol. 2, p. 467). Mais surtout ils diffèrent dans leur action sur l'angiogenèse. Tous deux sont angiogéniques in vivo; le TGF $\beta$ en particulier provoque, dans la cornée du rat et du lapin la formation d'un infiltrat leucocytaire avec néovascularisation intense. En revanche, les deux facteurs s'opposent dans leur action in vitro: le TGF $\alpha$ stimule la prolifération des cellules endothéliales en culture. Au contraire, le TGF $\beta$ inhibe cette prolifération, ce qui le rapproche d'autres protéines inhibitrices comme l'hormone antimüllérienne.

Les TNF (tumor necrosis factors) ont été récemment ajoutés à la panoplie des facteurs angiogéniques. Ils sont génétiquement étroitement liés entre eux et au complexe d'histocompatibilité chez l'homme [6]. C'est actuellement le $\mathrm{TNF} \alpha$ qui est le mieux connu [7]. Plus encore que pour les précédents, son nom est arbitraire, tant est diverse la palette de ses actions. Il possède d'ailleurs au moins un autre nom, celui de cachectine $\left(m / s n^{o} 1\right.$, vol. 2 , p. 49). Parmi ses activités multiples $\left(m / s n^{\circ} 2\right.$, vol. 3 , p.112) deux ont été étudiées en détail : la propriété qui lui a valu son nom - son pouvoir cytotoxique sur certaines tumeurs - pouvant aboutir à la régression complète; l'autre, in vitro, est sa puissante action inhibitrice sur la prolifération des cellules endothéliales en culture, agis- 
Tableau

\begin{tabular}{|lcc|}
\hline & Angiogenèse & Prolifération \\
\hline FGF a & + & + \\
FGF b & + & + \\
Angiogénine & + & 0 \\
TGF $\alpha$ & + & + \\
TGF $\beta$ & + & - \\
TNF $\alpha$ & + & - \\
\hline
\end{tabular}

$+=$ activation $;-=$ inhibition $; 0=$ absence d'effet.

sant comme un antagoniste de FGF C'est dire la surprise qu'ont provoquée les observations de deux groupes [8, 9] démontrant in vivo (comme pour le TGF $\beta$ ) une action tout à fait opposée: à des doses de l'ordre du nanogramme, dans la cornée du rat et du lapin comme dans la membrane chorio-allantoïque du poulet, le TNF stimule la néovascularisation ; la réponse inflammatoire suggère que cette propriété pourrait résulter d'une infiltration leucocytaire. Le TNF $\beta$, voisin mais différent du TNF $\alpha$, partage avec lui ces propriétés angiogéniques et probablement inflammatoires [10].

Les facteurs angiogéniques forment un ensemble complexe, et, pour une part, paradoxal. Le Tableau 1 montre la comparaison entre les actions in vivo et in vitro de ces polypeptides. L'hypothèse qui pourrait le mieux rendre compte de ces discordances est la suivante[1]: certains facteurs auraient directement pour cible la cellule vasculaire endothéliale; ils exerceraient la même action in vivo et in vitro; ce serait le cas des FGF et du TGF $\alpha$. Les autres facteurs auraient un mode d'action indirect, et tout le problème est de l'identifier. Une des voies les plus probables est l'activation des macrophages : ceuxci sécrètent alors des facteurs angiogéniques et notamment du TNF.

La question que l'on peut se poser est de savoir pourquoi, avec tant de facteurs susceptibles de la provoquer, l'angiogenèse est-elle habituellement quiescente en l'absence de causes déclenchantes. Plusieurs éléments concourent à cet équilibre : certains facteurs, directement actifs comme $m / s n^{\circ} 5$ vol. 1. mai 88 ladies angiogéniques ». A l’opposé, il pourrait exister des situations d'insuffisance de l'angiogenèse, à l'origine de retards de la guérison de blessures ou de fractures.

Ces notions devraient amener à envisager la thérapeutique de certaines affections sous un angle nouveau, conduisant à des tentatives d'activation ou d'inhibition de la croissance des capillaires. Dans le domaine du cancer, on aurait une chance de faire régresser une tumeur si on pouvait l'empêcher de déployer un système vasculaire suffisant pour permettre sa croissance.

les FGF, sont séquestrés à l'intérieur de la cellule, d'autres sont sous forme d'un précurseur inactif. De plus, des inhibiteurs, dont certains ne sont pas encore identifiés, existent; parmi eux figurent des dérivés du cortisol et des molécules proches de l'héparine. On pourrait considérer l'ensemble des facteurs de l'angiogenèse comme un système comparable à celui de la coagulation, normalement au repos mais prêt à se déclencher à la moindre alerte[1]. Ceci nous amène au dernier point, le plus important pour un médecin. Quel est le rôle de ces facteurs en pathologie? C'est dans les tumeurs que le processus angiogénique est le plus impressionnant, il ne semble cependant pas y avoir de différences qualitatives ni dans la nature chimique des peptides produits ni dans la morphologie des capillaires néoformés. La différence est que, dans les tumeurs, l'angiogenèse ne subit aucune auto-limitation : elle se poursuit jusqu'à l'élimination de la tumeur ou la mort de l'hôte, aucun des mécanismes normaux de contrôle n'étant mis en jeu. Le contraste est frappant avec, par exemple, le processus de guérison des blessures, où les macrophages cessent d'entretenir l'angiogenèse dès que de nouveaux capillaires ont colonisé la blessure. Mais, entre ces deux extrêmes, on entrevoit le rôle d'une persistance de l'angiogenèse dans des maladies qui ne semblent avoir aucun lien entre elles: affections rétiniennes comme l'artériopathie diabétique, l'arthrite rhumatoïde ou des hémangiomes; Folkman [1] propose de grouper ces maladies sous le vocable de «ma-
Jean-Claude Dreyfus

\section{RÉFÉRENCES}

1. Folkman J, Klagsbrun M. Angiogenic fac tors. Science $1987 ; 235: 442-7$.

2. Folkman J, Klagsbrun M. A family of angiogenic peptides. Nature 1987; 329 : 6712 .

3. Shing Y, Folkman J, Sullivan R, et al. Heparin affinity: purification of a tumor derived capillary endothelial cell growth factor. Science 1984; 223 : 1296-9.

4. Schreiber AB, Winkler ME, Derynck R Transforming growth factor $\alpha$ a more potent angiogenic mediator than epidermal growth factor. Science 1986 ; 232 : 1250-3.

5. Derynck R, Jarrett JA, Chen EY, et al Human transforming growth factor- $\beta$ complementary DN $\mathrm{A}$ sequence and expression in normal and transformed cells. Nature 1985 ; $316: 701-5$

6. Spies T, Morton CC, Nedospasof SA, et al. Genes for the tumor necrosis factors $\alpha$ and $\beta$ are linked to the human major histocompatibility complex. Proc Natl Acad Sci L'SA 1986: $83: 8699-702$.

7. Pennica D, Nedwin GE, Hayflick JS, et al. Human tumor necrosis factor: precursor structure. expression and homology to lym photoxin. Nature $1984: 312: 724-9$

8. Frater-Schrö̈ler M, Risau W, Hallmann R, Gauthsch P. Böhlen P. Tumor necrosis factor type $\alpha$, a potent inhibitor of endothelial cell growth in vitro, is angiogenic in vivo. Proc Natl Acad Sci L'SA 1987 ; 84 : 5277-81.

9. Leibovich SJ, Polverini, Shepard HM, et al. Macrophage-induced angiogenesis is mediated by tumor necrosis factor- $\alpha$. Nature $1987 ; 329: 630-2$.

10. Roberts AB, Sporn MB, Assoian RK Transforming growth factor type $\beta$ : rapid induction of fibrosis and angiogenesis in vivo and stimulation of collagen formation in vitro. Proc Natl Acad Sci USA 1986; 83 : 416771 . 